\title{
Genome modification of CXCR4 by Staphylococcus aureus Cas 9 renders cells resistance to HIV-1 infection
}

\author{
Qiankun Wang ${ }^{1 \dagger}$, Shuliang Chen ${ }^{1,2^{*} \dagger}$, Qiaoqiao Xiao ${ }^{1}$, Zhepeng Liu' ${ }^{1}$, Shuai Liu', Panpan Hou ${ }^{3}$, Li Zhou ${ }^{4}$, \\ Wei Hou', Wenzhe Ho ${ }^{1}$, Chunmei $\mathrm{Li}^{1,5}, \mathrm{Li} \mathrm{Wu}^{2}$ and Deyin Guo ${ }^{1,5^{*}}$ (1)
}

\begin{abstract}
Background: The CRISPR/Cas9 system has been widely used for genome editing in mammalian cells. CXCR4 is a co-receptor for human immunodeficiency virus type 1 (HIV-1) entry, and loss of CXCR4 function can protect cells from CXCR4 (X4)-tropic HIV-1 infection, making CXCR4 an important target for HIV-1 gene therapy. However, the large size of the CRISPR/SpCas9 system presents an obstacle to its efficient delivery into primary CD4 ${ }^{+} \mathrm{T}$ cells. Recently, a small Staphylococcus aureus Cas9 (SaCas9) has been developed as a genome editing tool can address this question. Therefore, it provides a promising strategy for HIV-1 gene therapy if it is used to target CXCR4.

Results: Here, we employed a short version of Cas9 from Staphylococcus aureus (SaCas9) for targeting CXCR4. We demonstrated that transduction of lenti-virus expressing SaCas9 and selected single-guided RNAs of CXCR4 in human $\mathrm{CD}^{+} \mathrm{T}$ cell lines efficiently induced the editing of the CXCR4 gene, making these cell lines resistant to X4-tropic HIV-1 infection. Moreover, we efficiently transduced primary human $\mathrm{CD} 4^{+} \mathrm{T}$ cells using adeno-associated virus-delivered CRISPR/SaCas 9 and disrupted CXCR4 expression. We also showed that CXCR4-edited primary $\mathrm{CD}^{+}{ }^{\mathrm{T}}$ cells proliferated normally and were resistant to HIV-1 infection.
\end{abstract}

Conclusions: Our study provides a basis for possible application of CXCR4-targeted genome editing by CRISPR/ SaCas9 in HIV-1 gene therapy.

Keywords: CXCR4, HIV-1, Primary CD4 ${ }^{+} \mathrm{T}$ cells, Adeno-associated virus, CRISPR/SaCas9

\section{Background}

Human immunodeficiency virus type 1 (HIV-1) is the causative agent of acquired immunodeficiency syndrome (AIDS). According to WHO, over 2.1 million people were newly infected with HIV-1 in 2015 with an estimated 1.1 million people dying of AIDS-related illnesses in the same year, and currently over 36.7 million people worldwide are HIV-1-positive (http://www.who.int/en/). The

\footnotetext{
*Correspondence: chen-shuliang@whu.edu.cn; guodeyin@mail.sysu.edu.cn

${ }^{\dagger}$ Qiankun Wang and Shuliang Chen contributed equally to this work ${ }^{1}$ School of Basic Medical Sciences, Wuhan University, Wuhan 430072, People's Republic of China

${ }^{5}$ School of Medicine (Shenzhen), Sun Yat-sen University, Zhongshan Erlu 74, Yuexiu District, Guangzhou 510080, People's Republic of China Full list of author information is available at the end of the article
}

use of antiretroviral therapy (ART) limits HIV-1 replication to an undetectable level, thus improving the health and life expectancy of HIV-1 infected individuals. However, ART does not represent a complete cure for HIV-1 infection as it is not effective against a persistent latent viral reservoir and has several side effects $[1,2]$. Thus, new curative approaches with limited drawbacks and higher efficiency for the treatment of HIV/AIDS are urgently needed.

HIV-1 entry into target cells is initiated by binding of the viral envelope protein gp120 to the primary receptor CD4 and a major co-receptor, either CXCR4 or CCR5 $[3,4]$. CCR5 is the co-receptor required for the entry of CCR5 (R5)-tropic strains of HIV-1, while CXCR4 (X4)-tropic viral strains are dependent on the CXCR4 co-receptor. Individuals with a naturally homozygous 
CCR5 432 deletion are highly resistant to HIV-1 infection $[5,6]$. Furthermore, previous studies reported a functional cure of HIV-1 infection when an AIDS patient with leukemia received a bone-marrow transplant from a tissue-matched donor with homozygous CCR $5 \triangle 32$ mutation $[7,8]$. Thus, the co-receptor CCR5 has been the major target for genome editing against HIV-1 infection. However, X4-tropic HIV-1 strains emerge in nearly a half of the patients initially infected with R5-tropic HIV-1 and their emergence is associated with a faster disease progression $[9,10]$. Therefore, CXCR4 should be considered another important target for anti-HIV-1 gene therapy.

Over the last decade, novel genome-editing methods that utilize nucleases have been developed, including zinc finger nucleases (ZFNs) [11], transcription activator like-effector nucleases (TALENs) [12] and clustered regularly interspaced short palindromic repeats (CRISPR)/ CRISPR-associated nuclease (Cas9) [13, 14]. Disruption of CXCR4 by ZFN-mediated genome editing conferred resistance to X4-tropic HIV-1 in several studies. Wilen et al. showed that disruption of CXCR4 with ZFNs conferred resistance of human $\mathrm{CD}^{+} \mathrm{T}$ cells to X4-tropic HIV-1 strains [15]. Yuan et al. showed that disruption of CXCR4 with ZFNs in human $\mathrm{CD} 4^{+} \mathrm{T}$ cells provided protection from HIV-1 infection in tissue cultures and in NSG mice [16]. Using the same approach, Didigu et al. showed that simultaneous genetic modification of CCR5 and $C X C R 4$ in primary human $\mathrm{CD}^{+} \mathrm{T}$ cells rendered cells resistant to infection with R5- and X4-tropic HIV-1 strains in vitro and in vivo [17].

CRISPR/Cas9 offers several advantages over conventional ZFN and TALEN, such as simple to design, easy to use and multiplexing [18]. Hultquist et al. edited the CXCR4 or CCR5 gene in primary $\mathrm{CD} 4^{+} \mathrm{T}$ cells by electroporation of CRISPR/Cas9 ribonucleoproteins [19]. We previously showed that the first generation of CRISPR/SpCas9 system was able to disrupt CXCR4 in primary human $\mathrm{CD} 4^{+} \mathrm{T}$ cells and generate HIV-1 resistance [20]. However, the large size of the CRISPR/ SpCas9 system restricts its efficient delivery into primary $\mathrm{CD} 4^{+} \mathrm{T}$ lymphocytes. Li et al. used a chimeric adenovirus as a vector for the delivery of CRISPR/ SpCas9, which resulted in the efficient silencing of CCR5 and, thus, HIV-1 resistance in primary $\mathrm{CD}^{+} \mathrm{T}$ cells [21]. In contrast, Wang et al. showed that lentiviral vectors expressing $\mathrm{SpCas} 9$ and sgRNA efficiently disrupt the $C D 4, C C R 5$ and $C X C R 4$ genes in transduced human $\mathrm{CD}^{+} \mathrm{T}$ cell line, but not in primary human $\mathrm{CD} 4^{+} \mathrm{T}$ cells [22].

One of the major challenges for CRISPR/Cas9 gene editing technologies is the delivery efficiency of the large gene cassettes. Viral vectors that including lentivirus, adenovirus, adeno-associated virus (AAV) are potential delivery vehicles for CRISPR/Cas9 components [23, 24]. AAV capsids can package less than $4.7 \mathrm{~kb}$ of singlestranded DNA, leaving little room for inserting other genetic elements when adopting the widely used Cas9 from Streptococcus pyogenes (SpCas9, $4.2 \mathrm{~kb}$ ). The Cas9 from Staphylococcus aureus (SaCas9) is $1 \mathrm{~kb}$ shorter than SpCas9 and thus can be packaged into the AAV genome together with a sgRNA gene expression cassette. Moreover, SaCas9 has a longer protospacer-adjacent motif (PAM) of 5'-NNGRRT-3' sequence compared to SpCas9 PAM of $5^{\prime}$-NGG-3'. These features allow easier delivery to cells by AAV expression vectors, and higher sequence specificity, which would be more desirable for therapeutic applications [25]. AAV-mediated SaCas9/sgRNA could be used to excise the integrated HIV-1 genome in vivo $[26,27]$. Using AAV as a gene therapy vector has many advantages over other commonly used recombinant viral vectors, such as low toxicity, sustained gene expression, safe and efficient delivery [28]. Recent studies reported that use of AAV6 in combination with electroporation of nuclease mRNA results in efficient homologous recombination repair (HDR) -mediated genome editing in transduced primary $\mathrm{T}$ cells and $\mathrm{CD} 34^{+}$hematopoietic cells [29-31]. We thus sought to deliver the CRISPR/SaCas9 into primary $\mathrm{CD} 4^{+} \mathrm{T}$ cells using an AAV6 vector.

In the present study, we selected two SaCas9/sgRNAs with high specificity to target CXCR4 after screening of 12 sgRNAs in HEK293T cell lines by using lentiviral vectors. By utilizing an AAV vector, we demonstrated the efficacy of CXCR4 editing and established HIV-1 resistance in primary $\mathrm{CD} 4^{+} \mathrm{T}$ cells.

\section{Results}

\section{Disruption of CXCR4 in GHOST-X4 and TZM-bl cells} by lentiviral vector-mediated transduction renders cells resistant to HIV-1 infection

To silence CXCR4 expression, we designed 12 sgRNAs that target different sites within the CXCR4 exon 2 and CCR5 sgRNA as the negative control (Additional file 1: Table S1). In order to select the efficient target sites, we modified the lentiCRISPR v2 plasmid by replacing the SpCas9 with SaCas9, and cloned the sgRNAs into the vector (Fig. 1a). We first tested the ability of our modified lentiCRISPR/SaCas9 gene editing system to disrupt CXCR4 in HEK293T cells. Three days after the transfection, we tested the on-target efficacy of the SaCas9/sgRNAs using a T7E1 assay, which cleaves DNA at distorted duplexes caused by mismatches. The result showed that the 1100-bp PCR amplicon was cleaved by sgRNA \#4, \#8, \#9 \#10 and \#12 guided SaCas9 while the negative sgRNA (sg-CCR5) guided no detectable editing (Additional file 2: Figure S1). We also observed that sgRNA \#8 and 
a

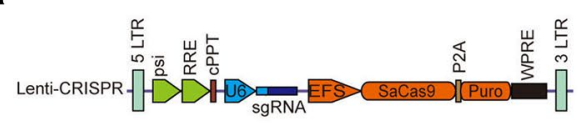

C

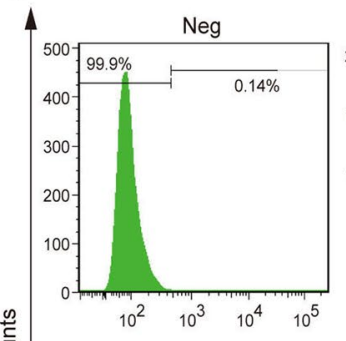

仓ั)
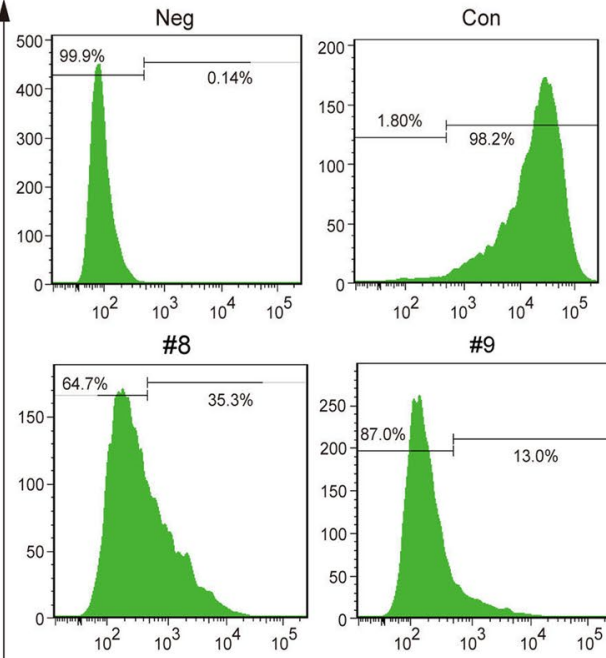

\#9

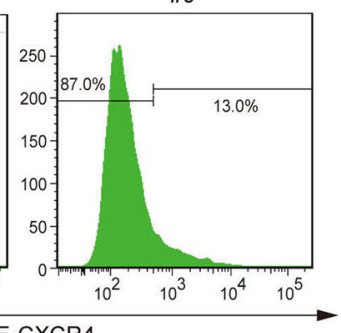

PE-CXCR4 b

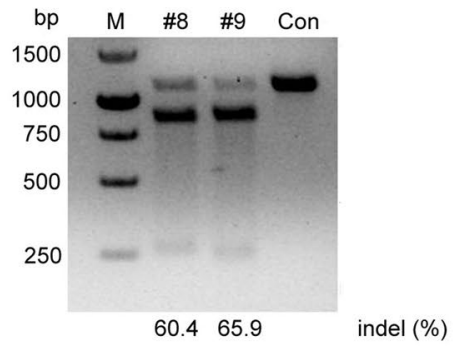

f

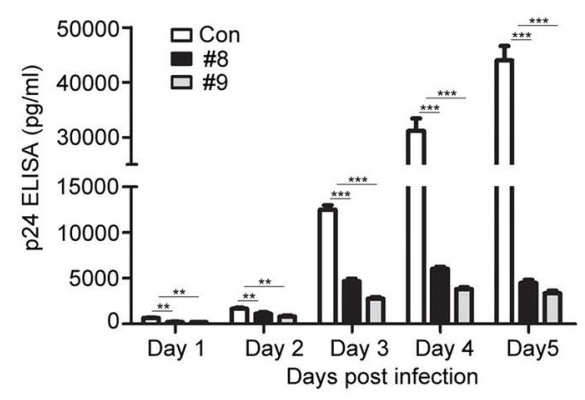

d

\#8

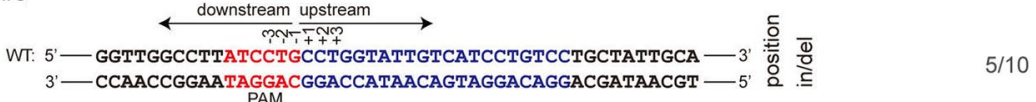

$$
\begin{aligned}
& \text { GGTTGGCCTTATCCTGCCTGGTATTGTCATC---TCCTGCTATTGCA } \quad(+16,-3) \quad 1 / 10 \\
& \text { GGTTGGCCTTATCCTGCCT---------AтCCTGTCCTGCTATTGCA } \quad(+4,-7) \quad 1 / 10 \\
& \text { GGTTGGCCTTATCCTGCCTGGTATTGTCATCCTGTCCTGCTATTGCA }(+6,+1) \quad 1 / 10 \\
& \text { GGTTGGCC-----------------TCATCCTGTCCTGCTATTGCA } \quad(-8,-18) \quad 1 / 10 \\
& \text { GGTTGGC-----------GGTATTGTCATCCTGTCCTGCTATTGCA } \quad(-9,-12) \quad 1 / 10 \\
& \text { \#9 } \\
& \text { WT: 5' - TGGTATTGTCATCCTGTCCTGCTATTGCATTATCATCTCCAAGCTGT - } 3 \text { ' } \\
& \text { 3' - ACCATAACAGTAGGACAGGACGATAACGTAATAGTAGAGGTTCGACA - 5' } \\
& \text { TGGTATTGTCATCCTGTC----TATTGCATTATCATCTCCAAGCTGT }
\end{aligned}
$$

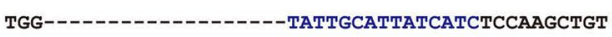

$$
\begin{aligned}
& \text { TGGTATTGTCATCCTG-CCTGCTATTGCATTATCATCTCCAAGCTGT } \\
& \text { TGGTATTGTCATCCTGTCCTGCTATTGCATTATCATCTCCAAGCTGT } \\
& 37 \mathrm{bp} \\
& \text {-TCCAAGCTGT } \\
& 250 \mathrm{bp} \\
& \text { ATTG AA GCTAC } \\
& \text { TGGTATTGTCATCCTGTCCTGCTATTGCATTATCATCTCCAAGCTGT } \quad(+3 /+5 /+7 /+10 /+13 /+15,1 / 10 \\
& \text { TAAT A }
\end{aligned}
$$

e

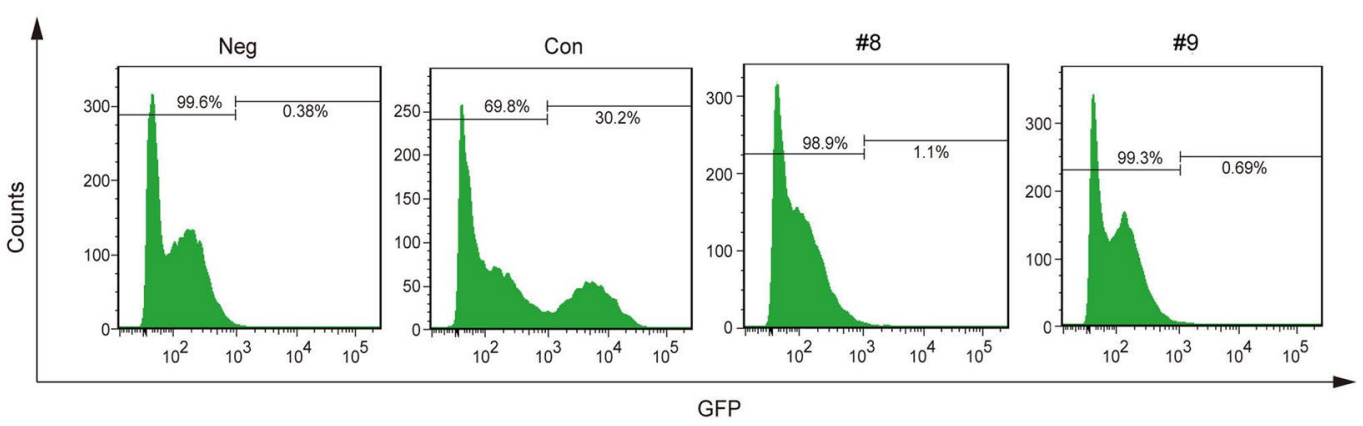




\section{(See figure on previous page.)}

Fig. 1 CXCR4 gene silencing by lentivirus mediated CRISPR/SaCas9 delivery protects GHOST-X4 cells from HIV-1 infection. a A schematic diagram of lentiviral transfer vectors containing CRISPR/SaCas9 components. LentiCRISPR v2 plasmid was modified by replacing the SpCas9 with SaCas9. Then, based on the SaCas9 PAM sequence 5'-NNGRRT-3', we designed, synthesized and cloned the CXCR4 sgRNAs into the vector using the Bsmb1. b CXCR4 gene disruption analysis in GHOST-X4 cells by the T7E1 cleavage assay. GHOST-X4 cells were transduced with lentiviruses (MOI of 40) in the presence of polybrene for $12 \mathrm{~h}$, and the genomic DNA was extracted and used as template to amplify a CXCR4 fragment (1 $100 \mathrm{bp})$. Con: lentiviral vectors expressing SaCas9 only, \#8 and \#9: lentiviral vectors expressing SaCas9/sgRNA \#8 and \#9. c Flow cytometry analysis of CXCR4 expression in lentivirus transduced GHOST-X4 cells. Neg, unstained cells. Con, \#8 and \#9 as samples described in (b) stained with anti-CXCR4-PE. d DNA sequences of CXCR4 of the transduced GHOST-X4 cells. PCR products were cloned into PGEM-T Easy vector and sequenced. The PAM sequences are lined and highlighted in red; the target sequences were shown in blue; deletions are indicated with ( - ) and insertions with (+). N/N indicates ratio of WT or mutations to total sequenced clones. e Flow cytometry analysis of transduced GHOST-X4 cells on GFP expression 3 days post HIV-1 NL4-3

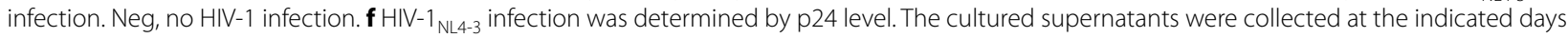
post infection, and HIV-1 p24 level was determined using a p24 ELISA kit. For (c and e), one representative out of three independent experiments is shown. For (f), the graph represents 3 independent infection experiments and error bars represent SEM. Statistical analysis determined using unpaired t-test $\left({ }^{* * *} P<0.001 ;{ }^{* *} P<0.01 ;{ }^{*} P<0.05\right)$

\#9 exhibited much higher efficiency than other sgRNAs (Additional file 2: Figure S1).

We subsequently tested the editing efficacy of the sgRNA \#8 and \#9 to disrupt CXCR4 in GHOST-X4 reporter cells, which express high levels of $\mathrm{CD} 4$ and CXCR4, and harbor a stable promoter element of long terminal repeat (LTR) -GFP reporter. Upon HIV-1 infection, viral Tat protein will bind to the LTR promoter and activate GFP expression [32]. The lentiCRISPR/SaCas9 vector was transduced into GHOST-X4 cells, and 3 days after the transduction, we tested the on-target efficacy of the SaCas9/sgRNAs using a T7E1 assay. The result showed that the 1100-bp PCR amplicon was efficiently cleaved into two fragments by SaCas9/sgRNAs \#8 and $\# 9$, leading to disruption of the CXCR4 gene (Fig. 1b). We also measured the expression of CXCR4 protein on the cell surface by flow cytometry. As shown in Fig. 1c, GHOST-X4 cells transduced with lentiviral vectors expressing SaCas9/sgRNA \#8 and \#9 became 64.7 and $87.0 \%$ negative for CXCR4 surface expression, respectively. In contrast, no reduction of CXCR4 expression was observed in cells transduced with empty vector (as a negative control). The sequencing results revealed that SaCas9/sgRNA \#8 and \#9 efficiently induced indel mutations in CXCR4 gene (Fig. 1d). We next tested the effect of the CXCR4 gene disruption on X4-tropic HIV-1 $1_{\mathrm{NL} 4-3}$ infection. We found that GHOST-X4 cells transduced with lentiviral SaCas9/sgRNA \#8 or \#9 became negative for GFP expression compared to control cells (Fig. 1e), indicating that lentiCRISPR/SaCas9-mediated genome modification of CXCR4 protects GHOST-X4 cells from HIV-1 infection. Furthermore, HIV-1 infection was determined by measurement of viral p24 in the culture supernatants. The levels of $\mathrm{p} 24$ released from the transduced cells were significantly decreased compared to the control cells (Fig. 1f).

Similarly, CXCR4 expression on another HIV-1 reporter cell line TZM-bl was also disrupted by SaCas9/ sgRNA \#8 and \#9 (Fig. 2a-c). TZM-bl cells are originally derived from HeLa cells expressing human CD4, CXCR4 and CCR5 as well as HIV-1 LTR-driven reporter genes including firefly luciferase [33]. SaCas9/sgRNA (\#8 and \#9) modified TZM-bl cells were infected with replication-competent $\mathrm{HIV}-1_{\mathrm{NL} 4-3}$ followed by luciferase reporter assay to quantify virus infection. Results revealed that $\mathrm{HIV}-1_{\mathrm{NL4}-3}$ infection in modified cells was largely decreased relative to the control cells, and $\mathrm{SaCas} 9 /$ sgRNAs \#9 showed higher disruption efficiency (Fig. 2d). Together, these data suggested that disruption of CXCR4 in HIV-1 reporter cell lines by lentivirus transduction renders cells resistant to X4-tropic HIV-1 infection.

\section{CXCR4 disruption by SaCas9/sgRNA in lentiviral vector-transduced Jurkat T cells inhibits HIV-1 infection} To test the effect of CRISPR/SaCas9-mediated disruption of CXCR4 in $\mathrm{CD} 4^{+} \mathrm{T}$ cell lines, we transduced Jurkat

(See figure on next page.)

Fig. 2 Disruption of CXCR4 in TZM-bl cells via lentiCRISPR/SaCas9 renders cells resistant to HIV-1 challenge. a CXCR4 gene disruption analysis in TZM-bl cells by the T7E1 cleavage assay. Assays were performed as in Fig. 1b. b Flow cytometry analysis of CXCR4 expression in lentivirus transduced TZM-bl cells. Assays were performed as in Fig. 1c. c DNA sequences of CXCR4 of the transduced TZM-bl cells. Assays were performed as in Fig. 1d. d Luciferase reporter assay to quantify HIV-1 infection level. Modified TZM-bl cells were infected with HIV-1 NL4-3 $_{3}(\mathrm{MOI}$ of 0.5 or 1$)$ for 6 h, then washed three times with PBS and cultured in complete DMEM medium for 3 days. Cells were collected and lysed in $100 \mu$ l of lysis buffer (Promega) for luciferase assay. For (b), one representative out of three independent experiments is shown. For (d), the graph represents 3 independent infection experiments and error bars represent SEM. Statistical analysis determined using unpaired t-test $\left.{ }^{* * *} P<0.001 ;{ }^{* *} P<0.01 ;{ }^{*} P<0.05\right)$ 
a

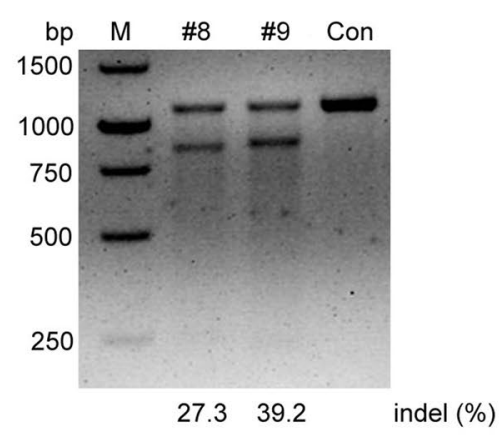

b

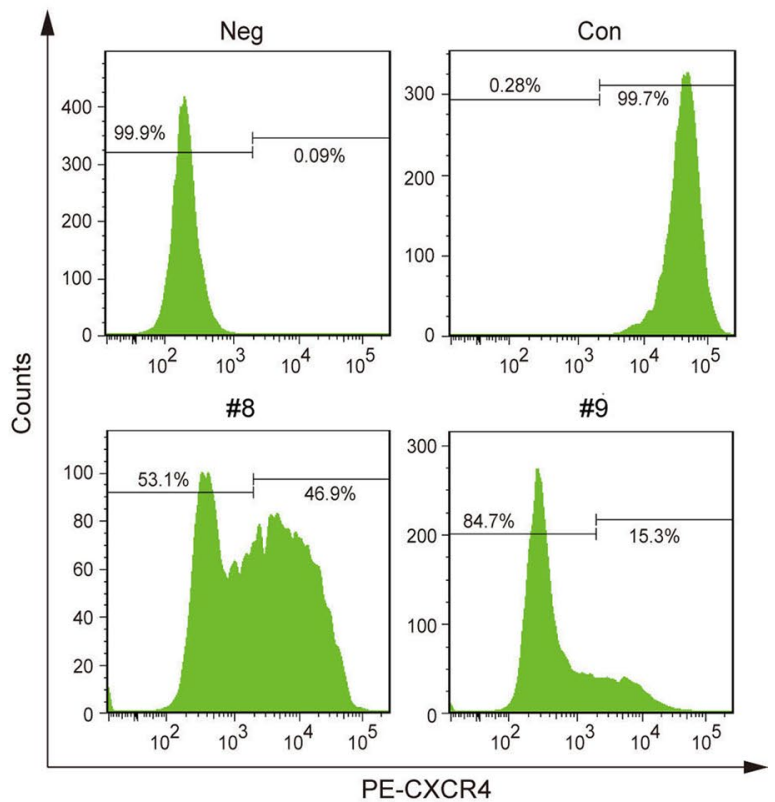

C

\#8

downstream upstream

WT: 5'-

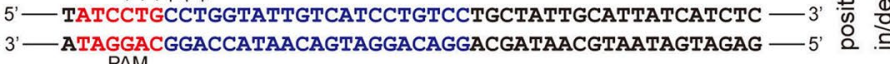

TATCCTGCCTGGTATTGTCATCCTG-CCTGCTATTGCATTATCATCTC

$(+17,-1)$

$4 / 10$

TATCCTGCCTGGTATTGTCATC-

$(+16,-102)$

$1 / 10$

TATCCTGCCTG

$(+5,-24)$

$1 / 10$

TATCCTGCCTGGTATTGTCATC-

$(+16,-24)$

$1 / 10$

TATCСTGCCTGG-

$(+6,-27)$

$1 / 10$

$(+15 /+17 /+18 /+19 \quad 1 / 10$

TATCCTGCCTGGTATTGTCATGCAGGA-TGATATTGCATTATCATCTC

$1+23 /+20$, mut/ -1$)$

\#9

WT: 5' - TTATCCTGCCTGGTATTGTCATCCTGTCCTGCTATTGCATTATCATCTCCAAGCT-3'

3'-AATAGGACGGACCATAACAGTAGGACAGGACGATAACGTAATAGTAGAGGTTCGA-5'

TTATCCTGCCTGGTATTGTCATCCTGTCCTG TTTGCATTATCATCTCCAAGCT

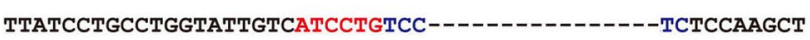

TTATCCTGCCTGGTATTGTCATC---TCCTGC---TGCATTATCATCTCCAAGCT

TTATCCTGCCTGGTATTG-

-TCCAAGCT

$84 \mathrm{bp}$

$5 / 10$

$(+6 /+8, \quad 1 / 10$

$\mathrm{mut} / \mathrm{mut})$

$(+4,-16) 2 / 10$

$\begin{array}{ll}(-3 /+7, & 1 / 10\end{array}$

$(-5 / 31, \quad 1 / 10$

d

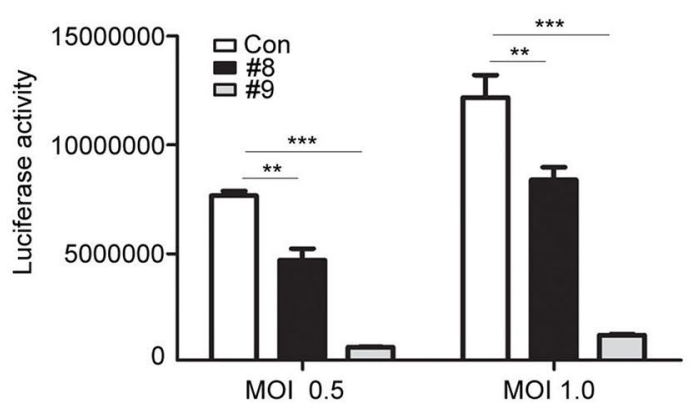


$\mathrm{T}$ cells with lentiviral vectors encoding SaCas9/sgRNA $\# 8$ and \#9. T7E1 assay and sequencing showed that the indels of CXCR4 gene were induced by SaCas9/sgRNA (Fig. 3a, c). We also performed flow cytometry to analyze CXCR4 protein expression and confirmed that cell surface CXCR4 expression was decreased by 62.2 and $73.6 \%$ using SaCas9/sgRNA \#8 and \#9, respectively (Fig. 3b). We further assessed the ability of SaCas9/sgRNAs to suppress HIV- $1_{\text {NL4-3 }}$ infection of Jurkat $\mathrm{T}$ cells by measuring viral p24 levels in culture supernatants at 1-7 days postinfection. Compared to the control cells, a significant decrease in p24 levels was observed in the Jurkat T cells treated with two SaCas9/sgRNAs (Fig. 3d). These data demonstrated that Jurkat $\mathrm{T}$ cells with disrupted CXCR4 gene by SaCas9/sgRNA are highly resistant to HIV-1 infection.

\section{CXCR4 editing protects primary $\mathrm{CD}^{+} \mathrm{T}$ cells from HIV-1 infection by AAV-CRISPR/SaCas9}

After successfully disrupting CXCR4 in cell lines by lentiviral vectors expressing SaCas9/sgRNAs, we attempted to deliver the CRISPR/SaCas9 components into primary $\mathrm{CD}^{+} \mathrm{T}$ cells. Despite our best efforts, we were not able to disrupt CXCR4 in primary human $\mathrm{CD}^{+} \mathrm{T}$ from multiple donors by lentiviral vectors expressing $\mathrm{SaCas} 9 /$ sgRNAs. We thus sought to deliver the CRISPR/SaCas9 into primary $\mathrm{CD}^{+} \mathrm{T}$ cells using an AAV vector. We constructed AAV vectors harboring the expression cassettes of SaCas9 and sgRNA \#8 or \#9, respectively (Fig. 4a). To evaluate the efficiency of AAV constructs for genome editing in primary $\mathrm{CD} 4^{+} \mathrm{T}$ cells, we performed the T7E1 assay and sequencing at 5 days post-transduction. Cells transduced with AAV-SaCas9/sgRNA \#8 and \#9 showed efficient cleavage of the CXCR4 gene compared with the control cells transduced with AAV expressing only SaCas9 (Fig. 4b, c). We also used electroporation to deliver the SaCas9/sgRNA \#8 and \#9 into CD4 ${ }^{+}$ $\mathrm{T}$ cells and observed disruption and indels in CXCR4 (Additional file 3: Figure S2). We next performed flow cytometry to analyze CXCR4 protein expression. CD4 ${ }^{+}$ $\mathrm{T}$ cells transduced with AAV vectors expressing SaCas9/ sgRNA \#8 and \#9 became 21.8 and $14.1 \%$ negative for CXCR4 surface expression, respectively. As a control, 8.07\% negative for CXCR4 expression was observed in cells transduced with empty vector (Fig. 4d). To examine whether CXCR4 silencing could affect cell viability, we performed a cell-viability assay at 1-7 days post-transduction and analyzed apoptosis upon AAV-mediated delivery of SaCas9/sgRNAs. The early apoptosis rate of SaCas9/sgRNA \#8 and \#9-transduced cells was 3.27 and $4.58 \%$, respectively, compared with $3.98 \%$ of control cells (Fig. 4f). These results revealed that CXCR4 editing did not significantly affect cell growth compared with control cells (Fig. 4e and f). Next, we assessed whether the editing of the CXCR4 could render cells resistant to replication-competent $\mathrm{HIV}-1_{\mathrm{NL} 4-3}$ infection. The AAVtransduced $\mathrm{CD} 4^{+} \mathrm{T}$ cells were infected with HIV-1 ${ }_{\mathrm{NL4}-3}$ and the culture supernatants were collected at $1-5$ days post-infection for p24 detection. The level of p24 from $\mathrm{CD}^{+}{ }^{+} \mathrm{T}$ cells transduced with SaCas9/sgRNA \#8 or \#9 was markedly decreased compared with that of cells transduced with empty vector at 3-5 days post-infection (Fig. 4g). These results suggested that the AAV-SaCas9/ sgRNA system can be used to mediate efficient editing of CXCR4 in primary $\mathrm{CD}^{+} \mathrm{T}$ cells and confer resistance to HIV-1 infection, while not affecting cell growth and viability.

\section{Specific targeting of CXCR4 by CRISPR/SaCas9 does not induce detectable off-target effects}

The off-target sites of CRISPR/SaCas9 were predicted and aligned with human genome using the online tool Cas-OFFinder (http://www.rgenome.net/cas-offinder/) [34]. We extracted 9 candidate off-target sites (Additional file 4: Table S2), and the sequences of these predicted offtarget sites were PCR amplified and subjected to T7E1 assay to identify the off-target activity. We did not detect any mutations using T7E1 assay (Additional file 5: Figure S3), indicating that CRISPR/SaCas9 showed high specificity of gene editing in our experiments.

\section{Discussion}

Currently available ART drugs do not provide an effective cure for HIV-1 infection. Gene therapy may represent a valuable alternative approach for AIDS treatment. One approach with ZFN-CCR5-modified autologous T cells of HIV-infected subjects is evaluated in an ongoing Phase 2 trial (SB-728) [35]. Although HIV-1 strains use

(See figure on next page.)

Fig. 3 Genome editing of CXCR4 in Jurkat T cells confers cell inhibition to HIV-1 infection by lentiCRISPR/SaCas9. a CXCR4 gene disruption analysis in Jurkat T cells by T7E1 cleavage assay. Assays were performed as in Fig. 1b. b Flow cytometry analysis of CXCR4 expression in lentivirus transduced Jurkat T cells. Assays were performed as in Fig. 1C. c DNA sequences of CXCR4 of the transduced Jurkat T cells. Assays were performed as in Fig. 1d. d HIV-1 p24 was detected in the Jurkat T cells treated with SaCas9/sgRNA. For (b), one representative out of three independent experiments is shown. For (d), the graph represents 3 independent infection experiments and error bars represent SEM. Statistical analysis determined using unpaired $t$ test $\left.{ }^{* *} P<0.001 ;{ }^{* *} P<0.01 ; * P<0.05\right)$ 
a

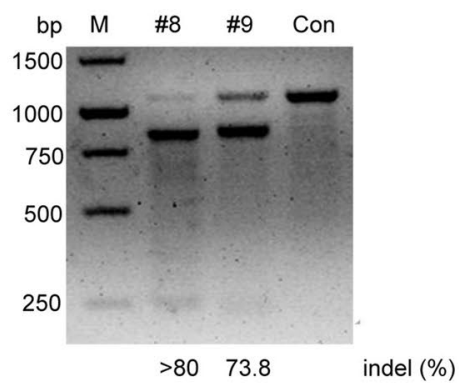

C

\#8 b

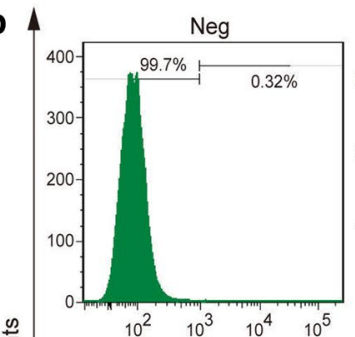

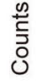
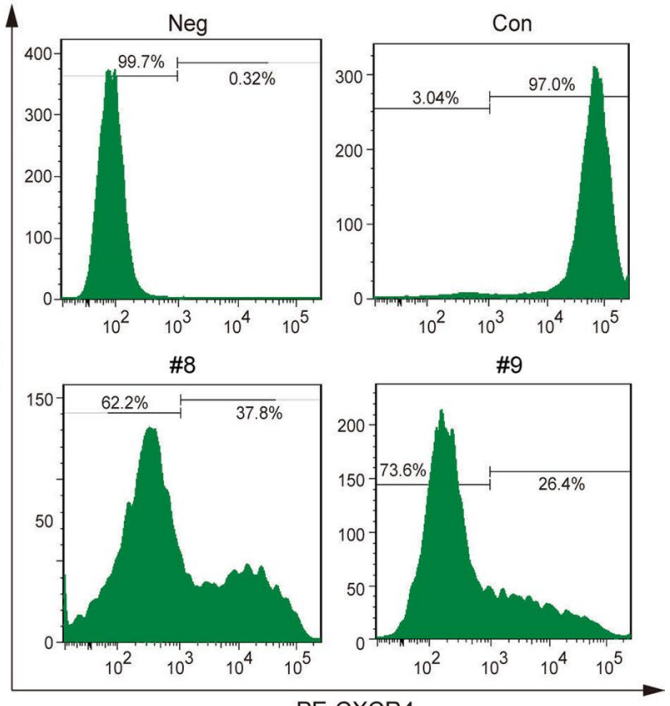

PE-CXCR4

d

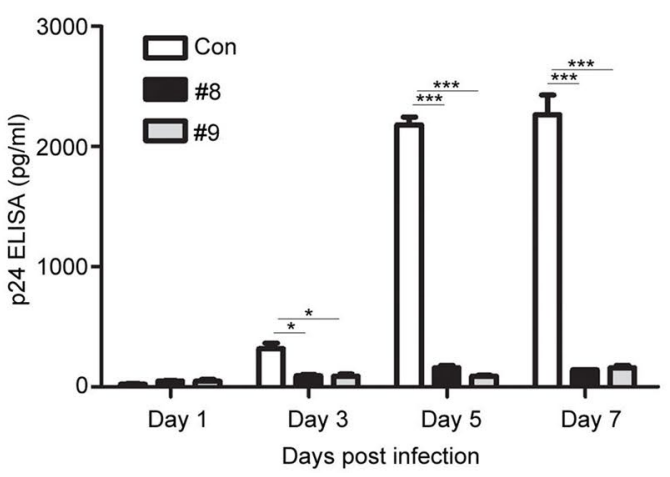




\section{a}

AAV-CRISPR- $-(1)-$ CMV SaCas9

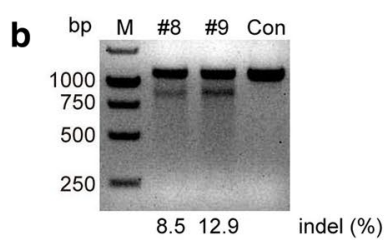

C \#8

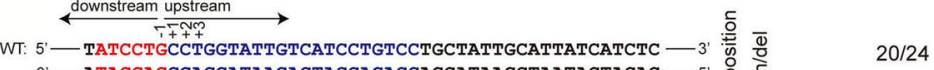

$$
\begin{aligned}
& \text { 3'- ATAGGACGGACCATAACAGTAGGACAGGACGATAACGTAATAGTAGAG - }-5 \text { ' } 8
\end{aligned}
$$

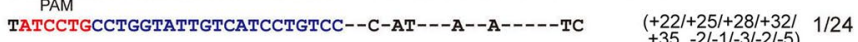

$$
\begin{aligned}
& \text { TATCCAGCCTGGTATTGTCATCCTGTCCTGCTATTGCATTATCATCTC } \quad(-2, \text { mut }) \quad 1 / 24 \\
& \text { TATCCTGCCTGGTATTGTCATCCTGTCCTGCTATTGCATTATCATCTC }(+4,+3) \quad 1 / 24 \\
& \text { TATCCTGCCTGGTATTGTCATCCTGTCCTGCTATTGCATTATCATCTC } \quad(+4,+7) \quad 1 / 24 \\
& \text { \#9 } \\
& \text { GAGGCCC }
\end{aligned}
$$

d
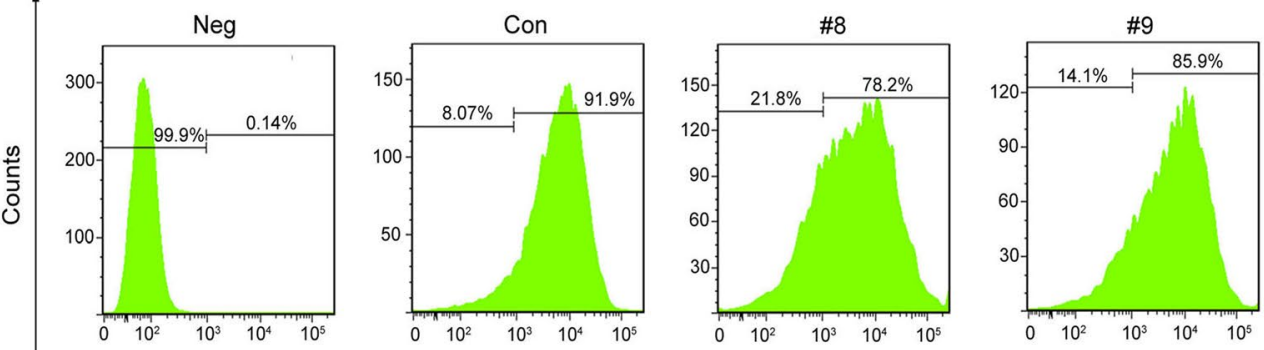

PE-CXCR4

e

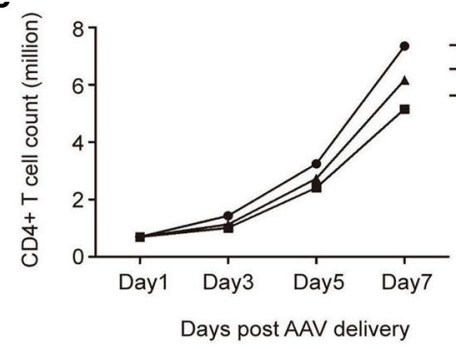

g

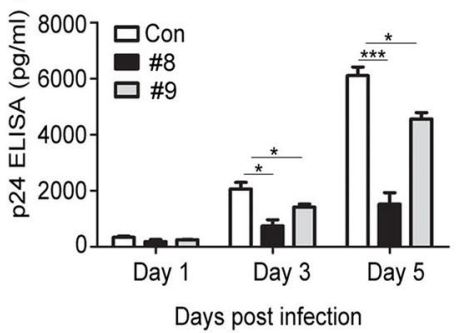

f
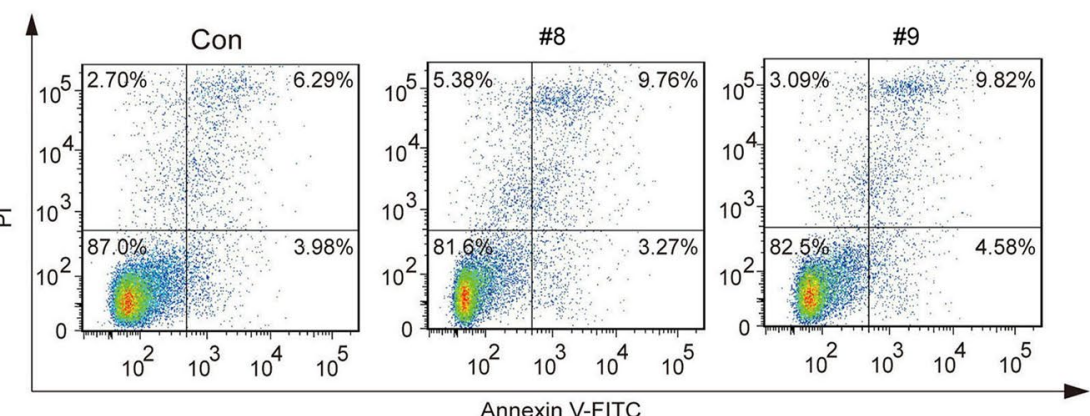

Annexin V-FITC 
(See figure on previous page.)

Fig. 4 AAV-mediated SaCas9/sgRNAs delivery suppresses HIV-1 infection in human primary CD4 ${ }^{+} \mathrm{T}$ cells. a A schematic diagram of AAV transfer vectors containing SaCas9 endonuclease and sgRNA. b T7E1 cleavage assay after 5 days post-transduction. c DNA sequences of CXCR4 of the AAV transduced $\mathrm{CD} 4^{+} \mathrm{T}$ cells. $\mathbf{d}$ Flow cytometry analysis of CXCR4 expression in AAV transduced CD4 ${ }^{+} \mathrm{T}$ cells. Assays were performed as in Fig. 1c. $\mathbf{e}$ $\mathrm{CD}^{+} \mathrm{T}$ cells counts at different times after transduction. CXCR4 disrupted cells continued to grow normally. $\mathbf{f}$ Flow cytometry analysis of apoptosis following AAV delivery SaCas9/sgRNA. $\mathbf{g}$ HIV-1 p24 was detected in the supernatants of the CD4 ${ }^{+}$T cells treated with AAV delivered SaCas9/sgRNA following HIV-1 $1_{\text {NL4-3 }}$ infection. For $(\mathbf{d}, \mathbf{e}$ and $\mathbf{f})$, one representative out of three independent experiments is shown. For $(\mathbf{g})$, the graph represents 3 independent infection experiments and error bars represent SEM. Statistical analysis determined using unpaired t-test $\left({ }^{* * *} P<0.001 ;{ }^{* *} P<0.01\right.$; $* P<0.05)$

CCR5 as a major coreceptor when establishing initial infections, CXCR4 is also a coreceptor for CXCR4-tropic HIV-1 infection in vivo $[9,10]$. Therefore, disruption of CXCR4 expression can complement CCR5-specific therapy against $\mathrm{HIV}-1$ infection.

In our previous report, the SpCas9 system has been successfully used to disrupt CXCR4 in GHOST-X4 and Jurkat $\mathrm{T}$ cells by lenti-SpCas9/sgRNA with efficiency of 76.5 and $45.1 \%$ respectively [20]. In the parental assay, the efficiency of lenti- SaCas9/sgRNA mediated CXCR4 editing of GHOST-X4 and Jurkat T cells are 87.0 and $73.6 \%$. These data suggested that SaCas9-mediated CXCR4 genome editing has more potential than SpCas9. Our previous results showed that editing of $C X C R 4$ in primary human $C D 4^{+} \mathrm{T}$ cells by delivery of SpCas9/sgRNA utilizing nucleofection generates HIV-1 resistance [20]. Although nucleofection is a direct delivery approach, its low efficacy and toxicity to primary cells restrict its application. In this study, we employed a short version of Cas9 from Staphylococcus aureus and AAV delivery system for targeting CXCR4. AAV can transduce both dividing and non-dividing cells without integrating into the host genome and shows low toxicity and sustained gene expression, while being safe in human gene therapy $[23,25]$. We confirmed that primary human $C D 4^{+} \mathrm{T}$ cells could be efficiently transduced and edited by AAV-delivered CRISPR/SaCas 9 targets CXCR4. Thus, our report provides an alternative approach for disrupting CXCR4 for HIV-1 therapy.

As CXCR4 is the receptor for CXCL12 (SDF-1) and has important consequence for $\mathrm{T}$ cell function and trafficking [36], we should pay attention to the safety and side effects before clinical application by targeting CXCR4 in HIV-1 gene therapy. However, studies showed that $\mathrm{T}$ lymphocytes appear to develop normally in CXCR4 knockout mice, suggesting that disruption of CXCR4 in $\mathrm{CD}^{+}{ }^{+} \mathrm{T}$ cells may be tolerable $[37,38]$. Although debatable, disruption of CXCR4 by ZFN in several studies demonstrated that CXCR4 is not essential for CD4 ${ }^{+} \mathrm{T}$ cell viability and function [15-17]. In our previous two reports, CXCR4 disruption by CRISPR-SpCas9 did not cause cells viability in CD4 ${ }^{+} \mathrm{T}$ cell $[20,39]$. In this study, we also showed that CXCR4-edited primary $\mathrm{CD}^{+} \mathrm{T}$ cells proliferated normally. In addition, we found that sgRNA \# 9 works better than sgRNA\#8 in primary cells, while opposite effect was observed in the cell lines. This probably resulted from various efficacies of expression and activity of promoter between EFS-NS (lenti-CRISPR/ Cas9) and CMV (AAV-CRISPR/Cas9) in different cells, or other underlying mechanisms [40].

\section{Conclusions}

In summary, our results demonstrated that CXCR4 disruption in $\mathrm{CD}^{+} \mathrm{T}$ cell lines using lentiviral vectors expressing $\mathrm{SaCas} 9 / \mathrm{sgRNAs}$ results in resistance to HIV-1 infection. Moreover, we showed that transducing AAV6 expressing SaCas9/sgRNAs in primary CD4 ${ }^{+} \mathrm{T}$ cells confers protection to HIV-1 infection. Together, this work represents a proof-of-principle study and provides a potential alternative approach for HIV-1/AIDS genetic therapy by targeting CXCR4 with AAV-deliver SaCas9/ sgRNAs.

\section{Methods}

\section{sgRNA design and plasmid construction}

Based on the SaCas9 PAM sequence 5'-NNGRRT-3', 12 gRNAs targeting CXCR4 exon 2 were designed and synthesized with 5'-CACC and 5'-AAAC overhangs. For lenti-SaCas9-CXCR4-gRNA plasmid, we modified the lentiCRISPR v2 plasmid (Addgene \#52961) by replacing the SpCas9 with SaCas9, and cloned the sgRNAs into the vector using the Bsmb1 (Fermentas). For AAVSaCas9-CXCR4-gRNA plasmid, sgRNAs were inserted into the PX601 plasmid (Addgene \#61591) digested with Bsa1 (Fermentas). Oligonucleotides for sgRNAs targeting CXCR4 are shown in Additional file 1: Table S1.

\section{Cell culture and transfection}

HEK293T cells, GHOST-X4 cells, TZM-bl and Jurkat $\mathrm{T}$ cells were cultured as described previously [41]. HEK293T cells were transfected in 24-well plates using $1 \mu \mathrm{g}$ of plasmid DNA mixed with Polyethylenimine (PEI) (Polysciences) according to the manufacturer's instruction. 


\section{Primary $\mathrm{CD}^{+} \mathrm{T}$ cell isolation and electroporation}

Whole blood samples from healthy donors were purchased from the Wuhan Blood Center (Wuhan, China). Isolation of $\mathrm{CD}^{+}{ }^{+} \mathrm{T}$ cells was achieved using Miltenyi products (Miltenyi Biotech). Primary human $\mathrm{CD}^{+} \mathrm{T}$ cells were maintained in RPMI (Hyclone) supplemented with 10\% FBS (Gibco), 1\% penicillin/streptomycin (Hyclone), $20 \mathrm{ng} / \mathrm{ml} \mathrm{IL-2} \mathrm{(PeproTech)} \mathrm{and} \mathrm{activated} \mathrm{with}$ anti-CD3/CD28-beadscoated on culture plate (Biolegend). Cells were seeded at $1-2 \times 10^{6} / \mathrm{ml}$ and cultured in T25 flasks.

$\mathrm{CD}^{+} \mathrm{T}$ cells were electroporated using the Lonza Nucleofector 4D (program E0-115) and the P3 Primary Cell 4D-Nucleofector Kit (V4XP-3024). In brief, $5 \times 10^{6} \mathrm{CD}^{+} \mathrm{T}$ cells were collected and washed twice in PBS. The cells were resuspended in $100 \mu \mathrm{l}$ Nucleofector Solution with $5 \mu \mathrm{g}$ of AAV-CRISPR/SaCas9 plasmids respectively. The mixture was transferred into the Nucleocuvette vessel and electro-transfecte. Following electroporation, the cells were cultured in RPMI 1640 medium supplemented with $10 \%$ FBS.

\section{Virus production and infection}

Lentiviruses were produced by co-transfection of HEK293T cells with lenti-SaCas9-CXCR4-gRNA, psPAX2 and pMD2.G, followed by concentration of the virus stocks by ultracentrifugation. Lentiviruses and HIV- $1_{\mathrm{NL} 4-3}$ stocks were generated as described before [20]. GHOST-X4 cells, TZM-bl cells and Jurkat T cells were transduced with lentiviruses (MOI (multiplicity of infection) of 40) in the presence of $8 \mu \mathrm{g} / \mathrm{ml}$ polybrene (Sigma) for $12 \mathrm{~h}$, and then medium was changed. For Jurkat $\mathrm{T}$ cells, the 12 -well plate were spun at $1200 \mathrm{~g}$ for $1.5 \mathrm{~h}$ at $25^{\circ} \mathrm{C}$ then transferred to $37^{\circ} \mathrm{C} .36 \mathrm{~h}$ post-transduction, $1 \mu \mathrm{g} / \mathrm{ml}$ puromycin (Sigma) was added to kill untransduced TZM-bl and Jurkat $\mathrm{T}$ cells within 2 days. After removal of puromycin, transduced cells were further cultured until downstream analysis. AAV were purchased from Vigene Biosciences (Jinan, China). CD4 ${ }^{+}$ $T$ cells were cultured with IL-2 and activated with antiCD3/CD28-beads coated on culture plate for $24-36 \mathrm{~h}$ and then transduced with AAV vectors at $5 \times 10^{5}$ vector genome copy per cell in free-FBS media for $24 \mathrm{~h}$. The cells were washed 3 times with PBS then resuspended in fresh media.

\section{T7 endonuclease 1 (T7E1) cleavage assay and DNA sequencing}

Genomic DNA was extracted with the Blood and Cell Culture DNA Midi kit (TianGen, China) according to the manufacturer's instructions. The purified genomic DNA was used as a template to amplify a fragment of the CXCR4 gene using the specific primers. The fragment size was
1100 bp (Additional file 6: Table S3). The PCR products were digested with $\mathrm{T} 7$ endonuclease 1 (NEB) and resolved by $1.5 \%$ agarose gel electrophoresis. The densities of cut and uncut bands were calculated using Image $J$ software. We used Indel $(\%)=(1-\sqrt{1-\text { cut } / \text { uncut }+ \text { cut }}) \times 100 \%$ formulas to get the efficiency of cleavage activity. The T7EI cleaves DNA at distorted duplexes with an upper limit of disruption sensitivity 80\% [42]. To further analyze CXCR4 gene disruption, the above PCR products were cloned into pGEM-T Easy vector (Promega) and sequenced. The indels of the CXCR4 gene were identified by comparison with the wild-type CXCR4 sequence.

\section{Flow cytometry analysis}

To analyze cell surface expression of CXCR4, lentivirus transduced GHOST-X4 cells, TZM-bl cells and Jurkat $\mathrm{T}$ cells were incubated with a PE-conjugated mouse anti-human CXCR4 antibody (Biolegend) for $20 \mathrm{~min}$ on ice. Cells then were washed twice with PBS and analyzed by flow cytometry (FACS AriaIII, BD). To determine HIV-1 infection efficiency, modified GHOST-X4 cells were infected with $\mathrm{HIV}-1_{\mathrm{NL} 4-3}$ at an MOI of 1 for $6 \mathrm{~h}$, then washed three times with PBS and cultured in complete DMEM medium for 3 days. GHOST-X4 cells were collected and washed twice with PBS and assessed for GFP expression through flow cytometry (FACS ArialII, BD). Data were analyzed with FlowJo (TreeStar) software. To test cell viability, primary $\mathrm{CD} 4^{+}$ $\mathrm{T}$ cells were collected after 5 days post AAV transduction, and $5 \mu \mathrm{l}$ Annexin V-FITC and $5 \mu \mathrm{l}$ propidium iodide (PI) (BD) were added and incubated for $15 \mathrm{~min}$ at room temperature. The apoptosis of AAV transduced cells were determined by flow cytometry (FACS AriaIII, $\mathrm{BD})$.

\section{Luciferase activity assay and p24 detection by ELISA}

Modified TZM-bl cells were infected with $\mathrm{HIV}-1_{\mathrm{NL} 4-3}$ at an MOI of 0.5 or 1 for $6 \mathrm{~h}$, then washed three times with PBS and cultured in complete DMEM medium for 3 days. Cells were then washed once with PBS and lysed in $100 \mu \mathrm{l}$ of lysis buffer (Promega). Luciferase activity in $30 \mu \mathrm{l}$ of cell suspensions was measured by a BrightGlo Luciferase assay according to the manufacturer's instruction (Promega). The cultured supernatants were collected at indicated days post-infection, HIV-1 p24 was determined using a p24 ELISA kit (ZeptoMetrix).

\section{Off-target site analysis}

To analyze potential off-target mutations, we used an online platform Cas-OFFinder (http://www.rgenome. net/cas-offinder/) and allowed up to a $4 \mathrm{bp}$ mismatch. The primers for amplifying the off-target sites resulted in 600-bp amplicons centered near the off-target sites. 
The corresponding primers are listed in Additional file 6: Table S3. The modified Jurkat T cells genomic DNA was used as a template to amplify the off-target sites. The T7E1 assay was used to detect off-target cleavage.

\section{Statistical analysis}

Unpaired $t$ test was performed using GraphPad Prism version 5. ${ }^{*} P<0.05,{ }^{* *} P<0.01$, and ${ }^{* * *} P<0.001$ denote significant differences. All experiments were performed for at least three times.

\section{Additional files}

\section{Additional file 1: Table S1. Oligonucleotides for sgRNAs targeting} CXCR4 locus.

Additional file 2: Figure S1. CXCR4 gene disruption screening analysis in HEK293T cells by the T7E1 cleavage assay. HEK293T cells were transfected in 24-well plates using $1 \mu \mathrm{g}$ of plasmid DNA mixed with Polyethylenimine (PEI). Three days after the transfection, the genomic DNA was extracted and used as template to amplify a CXCR4 fragment (1100 bp). Neg: CCR5 sgRNA; Con: lentiviral vectors expressing SaCas9 only; \#1-\#12: lentiviral vectors expressing SaCas9/sgRNA \#1-\#12.

Additional file 3: Figure S2. (a) T7E1 cleavage assay after electroporation in $\mathrm{CD}^{+} \mathrm{T}$ cells. (b) DNA sequences of CXCR4 of electroporated CD4 ${ }^{+}$ T cells.

Additional file 4: Table S2. List of potential off-target sites for both 5' and $3^{\prime}$ sgRNAs.

Additional file 5: Figure S3. (a) Off-target analysis of CXCR4 (\#8) by T7E1 cleavage assay. (b) Off-target analysis of CXCR4 (\#9) by T7E1 cleavage assay.

Additional file 6: Table S3. Primers information for the study.

\section{Authors' contributions}

Conceived and designed the experiments: DG. Performed the experiments: QW, SC, QX, ZL, SL, PH, LZ, CL. Provided key suggestions: WH, WH, LW. Analyzed the data: DG, QW, SC. Wrote and revised the paper: QW, SC, LW, DG. All authors read and approved the final manuscript.

\section{Author details}

'School of Basic Medical Sciences, Wuhan University, Wuhan 430072, People's Republic of China. ${ }^{2}$ Center for Retrovirus Research, Department of Veterinary Biosciences, The Ohio State University, 1900 Coffey Road, Columbus, OH, USA. ${ }^{3}$ College of Life Sciences, Wuhan University, Wuhan, People's Republic of China. ${ }^{4}$ Animal Biosafety Level III Laboratory at the Center for Animal Experiment, Wuhan University, Wuhan, People's Republic of China. ${ }^{5}$ School of Medicine (Shenzhen), Sun Yat-sen University, Zhongshan Erlu 74, Yuexiu District, Guangzhou 510080, People's Republic of China.

\section{Acknowledgements}

We thank Dr. Feng Zhang (Broad Institute of MIT and Harvard, USA) for kindly providing the SaCas9 plasmid. We also thank Dr. Serena Bonifati for helping with revising the manuscript and providing important suggestions.

\section{Competing interests}

The authors declare that they have no competing interests.

\section{Funding}

This work was funded by the China National Special Research Program of Major Infectious Diseases (2014ZX10001003) and Hubei Provincial Science and Technology Innovation Team Grant (2012FFA043). DG is supported by the Hubei Province's Outstanding Medical Academic Leader Program. This work was also supported by the Natural Science Foundation of China (81401659) and China Postdoctoral Science Foundation (2015T80838 and 2014M560622) to SC. LW is supported by Grants (Al104483, Al127667, and Al120209) from the National Institutes of Health of the USA. This work was partially supported by the scholarship to SC from the China Scholarship Council.

\section{Publisher's Note}

Springer Nature remains neutral with regard to jurisdictional claims in published maps and institutional affiliations.

Received: 1 October 2017 Accepted: 8 November 2017

Published online: 15 November 2017

\section{References}

1. Anderson JL, Fromentin R, Corbelli GM, Ostergaard L, Ross AL. Progress towards an HIV cure: update from the 2014 international AIDS society symposium. AIDS Res Hum Retrovir. 2015;31(1):36-44.

2. Barre-Sinoussi F, Ross AL, Delfraissy JF. Past, present and future: 30 years of HIV research. Nat Rev Microbiol. 2013;1 1(12):877-83.

3. Feng Y, Broder CC, Kennedy PE, Berger EA. HIV-1 entry cofactor: functional cDNA cloning of a seven-transmembrane, $\mathrm{G}$ protein-coupled receptor. Science. 1996;272(5263):872-7.

4. Deng HK, Liu R, Ellmeier W, Choe S, Unutmaz D, Burkhart M, et al. Identification of a major co-receptor for primary isolates of HIV-1. Nature. 1996;381(6584):661-6.

5. Samson M, Libert F, Doranz BJ, Rucker J, Liesnard C, Farber CM, et al. Resistance to HIV-1 infection in caucasian individuals bearing mutant alleles of the CCR-5 chemokine receptor gene. Nature. 1996;382(6593):722-5.

6. Liu R, Paxton WA, Choe S, Ceradini D, Martin SR, Horuk R, et al. Homozygous defect in HIV-1 coreceptor accounts for resistance of some multiplyexposed individuals to HIV-1 infection. Cell. 1996;86(3):367-77.

7. Allers K, Hutter G, Hofmann J, Loddenkemper C, Rieger K, Thiel E, et al. Evidence for the cure of HIV infection by CCR5 $\triangle 32 / \Delta 32$ stem cell transplantation. Blood. 2011;117(10):2791-9.

8. Huetter G, Nowak D, Mossner M, Ganepola S, Muessig A, Allers K, et al. Long-term control of HIV by CCR $5 \Delta 32 / \Delta 32$ stem-cell transplantaion. New Engl J Med. 2009;360(7):692-8.

9. Connor RI, Sheridan KE, Ceradini D, Choe S, Landau NR. Change in coreceptor use correlates with disease progression in HIV-1-infected individuals. J Exp Med. 1997;185(4):621-8.

10. Huang W, Toma J, Stawiski E, Fransen S, Wrin T, Parkin N, et al. Characterization of human immunodeficiency virus type 1 populations containing CXCR4-using variants from recently infected individuals. AIDS Res Hum Retrovir. 2009;25(8):795-802.

11. Urnov FD, Rebar EJ, Holmes MC, Zhang HS, Gregory PD. Genome editing with engineered zinc finger nucleases. Nat Rev Genet. 2010;11(9):636-46.

12. Joung JK, Sander JD. TALENs: a widely applicable technology for targeted genome editing. Nat Rev Mol Cell Biol. 2013;14(1):49-55.

13. Cong L, Ran FA, Cox D, Lin S, Barretto R, Habib N, et al. Multiplex genome engineering using CRISPR/Cas systems. Science. 2013;339(6121):819-23.

14. Mali P, Yang L, Esvelt KM, Aach J, Guell M, DiCarlo JE, et al. RNA-Guided human genome engineering via Cas9. Science. 2013;339(6121):823-6.

15. Wilen CB, Wang J, Tilton JC, Miller JC, Kim KA, Rebar EJ, et al. Engineering HIV-resistant human CD4+ $T$ cells with CXCR4-specific zinc-finger nucleases. PLoS Pathog. 2011;7(4):e1002020.

16. Yuan J, Wang J, Crain K, Fearns C, Kim KA, Hua KL, et al. Zinc-finger nuclease editing of human Cxcr4 promotes HIV-1 CD4(+) T cell resistance and enrichment. Mol Ther. 2012;20(4):849-59.

17. Didigu CA, Wilen CB, Wang J, Duong J, Secreto AJ, Danet-Desnoyers GA, et al. Simultaneous zinc-finger nuclease editing of the HIV coreceptors CCR5 and CXCR4 protects CD4(+) T cells from HIV-1 infection. Blood. 2014;123(1):61-9.

18. Gaj T, Gersbach CA, Barbas CF. ZFN, TALEN, and CRISPR/Cas-based methods for genome engineering. Trends Biotechnol. 2013;31(7):397-405.

19. Hultquist JF, Schumann K, Woo JM, Manganaro L, McGregor MJ, Doudna J, et al. A Cas9 ribonucleoprotein platform for functional genetic studies of HIV-host interactions in primary human T Cells. Cell Rep. 2016;17(5):1438-52. 
20. Hou P, Chen S, Wang S, Yu X, Chen Y, Jiang M, et al. Genome editing of CXCR4 by CRISPR/Cas9 confers cells resistant to HIV-1 infection. Sci Rep. 2015:5:15577

21. Li C, Guan X, Du T, Jin W, Wu B, Liu Y, et al. Inhibition of HIV-1 infection of primary $\mathrm{CD} 4^{+} \mathrm{T}$-cells by gene editing of CCR5 using adenovirus-delivered CRISPR/Cas9. J Gen Virol. 2015;96(8):2381-93.

22. Wang W, Ye C, Liu J, Zhang D, Kimata JT, Zhou P. CCR5 gene disruption via lentiviral vectors expressing Cas 9 and single guided RNA renders cells resistant to HIV-1 infection. PLoS ONE. 2014;9(12):e115987.

23. Gori JL, Hsu PD, Maeder ML, Shen S, Welstead GG, Bumcrot D. Delivery and specificity of CRISPR-Cas9 genome editing technologies for human gene therapy. Hum Gene Ther. 2015;26(7):443-51.

24. Choi JG, Dang Y, Abraham S, Ma H, Zhang J, Guo H, et al. Lentivirus pre-packed with Cas9 protein for safer gene editing. Gene Ther. 2016;23(7):627-33.

25. Ran FA, Cong L, Yan WX, Scott DA, Gootenberg JS, Kriz AJ, et al. In vivo genome editing using Staphylococcus aureus Cas9. Nature. 2015:520(7546):186-91.

26. Kaminski R, Bella R, Yin C, Otte J, Ferrante P, Gendelman HE, et al. Excision of HIV-1 DNA by gene editing: a proof-of-concept in vivo study. Gene Ther. 2016;23(8-9):696.

27. Yin C, Zhang T, Qu X, Zhang Y, Putatunda R, Xiao X, et al. In vivo excision of HIV-1 provirus by saCas 9 and multiplex single-guide RNAs in animal models. Mol Ther. 2017;25(5):1168-86.

28. Mingozzi F, High KA. Immune responses to AAV vectors: overcoming barriers to successful gene therapy. Blood. 2013;122(1):23-36.

29. Sather BD, Romano Ibarra GS, Sommer K, Curinga G, Hale M, Khan IF, et al. Efficient modification of CCR5 in primary human hematopoietic cells using a megaTAL nuclease and AAV donor template. Sci Transl Med. 2015;7(307):307ra156

30. Wang J, DeClercq JJ, Hayward SB, Li PW, Shivak DA, Gregory PD, et al. Highly efficient homology-driven genome editing in human T cells by combining zinc-finger nuclease mRNA and AAV6 donor delivery. Nucleic Acids Res. 2016;44(3):e30.

31. Wang J, Exline CM, DeClercq JJ, Llewellyn GN, Hayward SB, Li PW, et al. Homology-driven genome editing in hematopoietic stem and progenitor cells using ZFN mRNA and AAV6 donors. Nat Biotechnol. 2015:33(12):1256-63.
32. Cecilia D, KewalRamani VN, O'Leary J, Volsky B, Nyambi P, Burda S, et al. Neutralization profiles of primary human immunodeficiency virus type 1 isolates in the context of coreceptor usage. J Virol. 1998;72(9):6988-96.

33. Derdeyn CA, Decker JM, Sfakianos JN, Zhang Z, O'Brien WA, Ratner L, et al. Sensitivity of human immunodeficiency virus type 1 to fusion inhibitors targeted to the gp41 first heptad repeat involves distinct regions of gp41 and is consistently modulated by gp120 interactions with the coreceptor. J Virol. 2001;75(18):8605-14.

34. Bae S, Park J, Kim JS. Cas-OFFinder: a fast and versatile algorithm that searches for potential off-target sites of Cas9 RNA-guided endonucleases. Bioinformatics. 2014;30(10):1473-5.

35. Wang CX, Cannon PM. Clinical applications of genome editing to HIV cure. AIDS Patient Care STDS. 2016;30(12):539-44.

36. Bleul CC, Fuhlbrigge RC, Casasnovas JM, Aiuti A, Springer TA. A highly efficacious lymphocyte chemoattractant, stromal cell-derived factor (SDF-1). J Exp Med. 1996;184(3):1101-9.

37. Nagasawa T, Hirota S, Tachibana K, Takakura N, Nishikawa S, Kitamura Y, et al. Defects of B-cell lymphopoiesis and bone-marrow myelopoiesis in mice lacking the CXC chemokine PBSF/SDF-1. Nature. 1996;382(6592):635-8.

38. Chung SH, Seki K, Choi BI, Kimura KB, Ito A, Fujikado N, et al. CXC chemokine receptor 4 expressed in T cells plays an important role in the development of collagen-induced arthritis. Arthritis Res Ther. 2010;12(5):R188.

39. Liu Z, Chen S, Jin X, Wang Q, Yang K, Li C, et al. Genome editing of the HIV co-receptors CCR5 and CXCR4 by CRISPR-Cas9 protects CD4 ${ }^{+} \mathrm{T}$ cells from HIV-1 infection. Cell Biosci. 2017:7:47.

40. Holehonnur R, Lella SK, Ho A, Luong JA, Ploski JE. The production of viral vectors designed to express large and difficult to express transgenes within neurons. Mol Brain. 2015;8:12

41. Liu Y, Zhou J, Pan J-A, Mabiala P, Guo D. A novel approach to block HIV-1 coreceptor CXCR4 in non-toxic manner. Mol Biotechnol. 2014:56(10):890-902

42. Guschin DY, Waite AJ, Katibah GE, Miller JC, Holmes MC, Rebar EJ. A rapid and general assay for monitoring endogenous gene modification. Methods Mol Biol. 2010;649:247-56.

\section{Submit your next manuscript to BioMed Central and we will help you at every step:}

- We accept pre-submission inquiries

- Our selector tool helps you to find the most relevant journal

- We provide round the clock customer support

- Convenient online submission

- Thorough peer review

- Inclusion in PubMed and all major indexing services

- Maximum visibility for your research

Submit your manuscript at www.biomedcentral com/submit
O Biomed Central 